\title{
Simpson Grade 2 Resection and Tumor Recurrence in Ventrally Located Spinal Meningiomas
}

\author{
Necati UCLER ${ }^{1}$, Batu HERGUNSEL ${ }^{2}$, Sait OZTURK², Serpil KOZAN³ ${ }^{3}$, Metin KAPLAN ${ }^{2}$ \\ ${ }^{1}$ Adiyaman University, Department of Neurosurgey, Adiyaman, Turkey \\ ${ }^{2}$ Firat University, Department of Neurosurgery, Elazig, Turkey \\ ${ }^{3}$ Malatya State Hospital, Pathology Laboratory, Malatya, Turkey
}

\section{ABSTRACT}

AIM: To evaluate postoperative clinical outcome and recurrence rates in cases with ventrally located spinal meningiomas who underwent Simpson grade 2 resection.

MATERIAL and METHODS: We evaluated eight cases (six females and two males; age, 28-86 years; mean age, 60 years) with ventrally located spinal meningioma that underwent surgical treatment in our clinic. The tumors were located in the thoracic region in seven cases and in the cervical region in one case. All cases underwent surgery, which was performed using a traditional posterior midline approach. Laminectomy was unilaterally extended. The case with cervically located tumor differed from the other cases in that a skull clamp was used and laminoplasty was performed.

RESULTS: The tumor was completely resected in all cases. Furthermore, in all cases, the tumor nidus that caused the thickening of the dura was cauterized using bipolar cautery and peeled off (Simpson grade 2). The pathological examination of the tumor specimens revealed typical grade 1 meningioma in all cases (fibropsammomatous type, $n=1$; meningothelial type, $n=3$; and psammomatous type, $n=4)$. Recurrence was not observed in any of the cases during the follow-up. None of the cases demonstrated postoperative neurological deterioration. At 1 month postoperatively, pain and motor deficit completely improved in all cases, with remarkable improvements of motor deficits in the early postoperative period.

CONCLUSION: Simpson grade 2 resection in ventrally located spinal meningiomas results in low complication rates and is satisfactory in terms of recurrence.

KEYWORDS: Meningioma, Simpson grade, Ventral spinal

\section{INTRODUCTION}

$\mathrm{S}$ pinal meningiomas account for $25 \%-46 \%$ of all intraspinal tumors (7), and are often located laterally to the spinal $\checkmark$ canal but are less often located ventrally or posteriorly $(3,8,10)$. Complete tumor resection is important to achieve good long-term results $(8,10)$. Tumor location and size are the major factors to consider in complete tumor resection. During surgery, it is important to note that the spinal cord can be stretched only to a certain extent. In general, Simpson grade 1 resection is recommended to minimize the recurrence rate in meningiomas. However, Simpson grade 1 resection is more difficult in cases with ventrally located spinal meningiomas than in those with posteriorly located meningiomas and increases the rate of complications $(11,12,15)$. The recurrence of spinal meningiomas is rare $(4,9,14)$. Therefore, the question "Is Simpson grade 1 resection necessary in ventrally located spinal meningiomas?" remains the current issue in neurosurgical practice (15).

In this study, we evaluated postoperative clinical cure and recurrence rates in cases with ventrally located spinal meningiomas that underwent Simpson grade 2 resection. 


\section{MATERIAL and METHODS}

We evaluated eight cases (six females and two males; age, 28-86 years; mean age, 60 years) with ventrally located spinal meningioma that underwent surgical treatment at our clinic (Table I). A 28-year-old patient's symptoms began in the early post-pregnancy period. All other patients were in their fifties or older.

The most frequent symptom was pain consistent with the location of the tumor, followed by hypoesthesia. Two other common symptoms and signs were pain and sensory deficit (Table II).

All tumors were radiologically diagnosed using magnetic resonance imaging $(\mathrm{MRI})$. The tumors were isointense in T1-weighted and hyperintense in T2-weighted MRI. The extramedullary localization and contrast enhancement of the tumors were typical of meningiomas. There was no tumor calcification in any of the cases. The tumors were located in the thoracic region in seven cases and in the cervical region in one case.

All cases underwent surgery, which was performed using a traditional posterior midline approach. Laminectomy was unilaterally extended. The case in which the tumor was cervically located differed from the other cases in that a skull clamp was used and laminoplasty was performed. No stabilization was performed, and no neuromonitoring device was used in any of the cases.

The cases were postoperatively followed-up for a mean period of 40 (range, 22-79) months. The postoperative neurological cure was followed-up in the early postoperative period at 1 and 3 months and at every 6 months thereafter. Radiological evaluation was followed-up using MRI in the early postoperative period at 3 months and once every 6 months thereafter. Data for recurrence and clinical cure were recorded.

\section{RESULTS}

The tumor was completely resected in all cases. Furthermore, in all cases, the tumor nidus that caused the thickening of

Table I: Cases of Ventral Intraspinal Meningioma the dura was cauterized using bipolar cautery and peeled off (Simpson grade 2). One case developed postoperative cerebrospinal fluid (CSF) fistula, which was successfully treated using closed continuous CSF drainage via a lumbar subarachnoid catheter. No other cases developed any complications.

The pathological examination of the tumor specimens revealed typical grade 1 meningioma in all cases (fibropsammomatous type, $n=1$; meningothelial type, $n=3$; and psammomatous type, $n=4)$. Recurrence was not observed in any of the cases during the follow-up period.

None of the cases demonstrated postoperative neurological deterioration. At 1 month postoperatively, pain and motor deficit completely improved in all cases, with remarkable improvement of motor deficits in the early postoperative period. Hypoesthesia in seven cases was completely resolved within 3 months postoperatively. However, hypoesthesia in the left arm persisted for 36 months postoperatively. None of the cases demonstrated pre- or postoperative sphincter dysfunction.

\section{DISCUSSION}

Spinal meningiomas are slow-growing benign lesions that are more commonly observed in females and during middle age. Psammomatous and meningothelial subtypes of spinal meningiomas are more prevalent. The most common symptoms of spinal meningiomas are pain and paresthesia (2$4,11,13,18)$. In this study, although we specifically evaluated cases with ventral localization that underwent Simpson grade 2 resection, the distributions of their age, sex, and histological subtypes were compatible with those of the cases described in the literature $(2-4,11,13,14,18)$. Our cases demonstrated the general characteristics of spinal meningioma, indicating that our series showed demographic features appropriate for the analysis.

Ventral localization is less common in spinal meningiomas $(1,3,8)$, and is a factor that causes difficulties during treatment. In addition to the conventional posterior midline approach, in ventrally located spinal meningiomas, laminectomy is extended

\begin{tabular}{ccccccc}
\hline Case no. & Age (years) & Sex & Localization & Histopathological type & Follow-up (months) \\
\hline 1 & 60 & F & T5 & Meningothelial & 24 \\
\hline 2 & 63 & M & T7 & Meningothelial & 47 & 23 \\
\hline 3 & 86 & F & T4 & Psammomatous & 40 \\
\hline 4 & 55 & F & T12 & Psammomatous & 22 \\
\hline 5 & 28 & F & T7 & Psammomatous & Meningothelial & 39 \\
\hline 7 & 48 & F & C4 & Fibropsammomatous & 79 \\
\hline
\end{tabular}

M: Male, F: Female, T: Thoracic, C: Cervical. 
Table II: Preoperative Clinical Signs

\begin{tabular}{lc}
\hline Signs & No (cases) \\
\hline Pain & 6 \\
\hline Sensory deficit & 5 \\
\hline Motor deficit & 4 \\
\hline Reflex changes & 4 \\
\hline Sphincter dysfunction & -
\end{tabular}

laterally to obtain better exposure. Despite this technique, a compressed and poorly perfused spinal cord is still vulnerable to surgical intervention, which potentially increases the rate of complications. Therefore, ventral localization is a risk factor in terms of surgical outcome and recurrence. In ventrally located meningiomas, a large tumor size, close proximity to the anterior spinal arterial system, and the presence of calcification increase the risk. Therefore, Simpson grade 1 resection is a subject of debate in ventrally located spinal meningiomas and

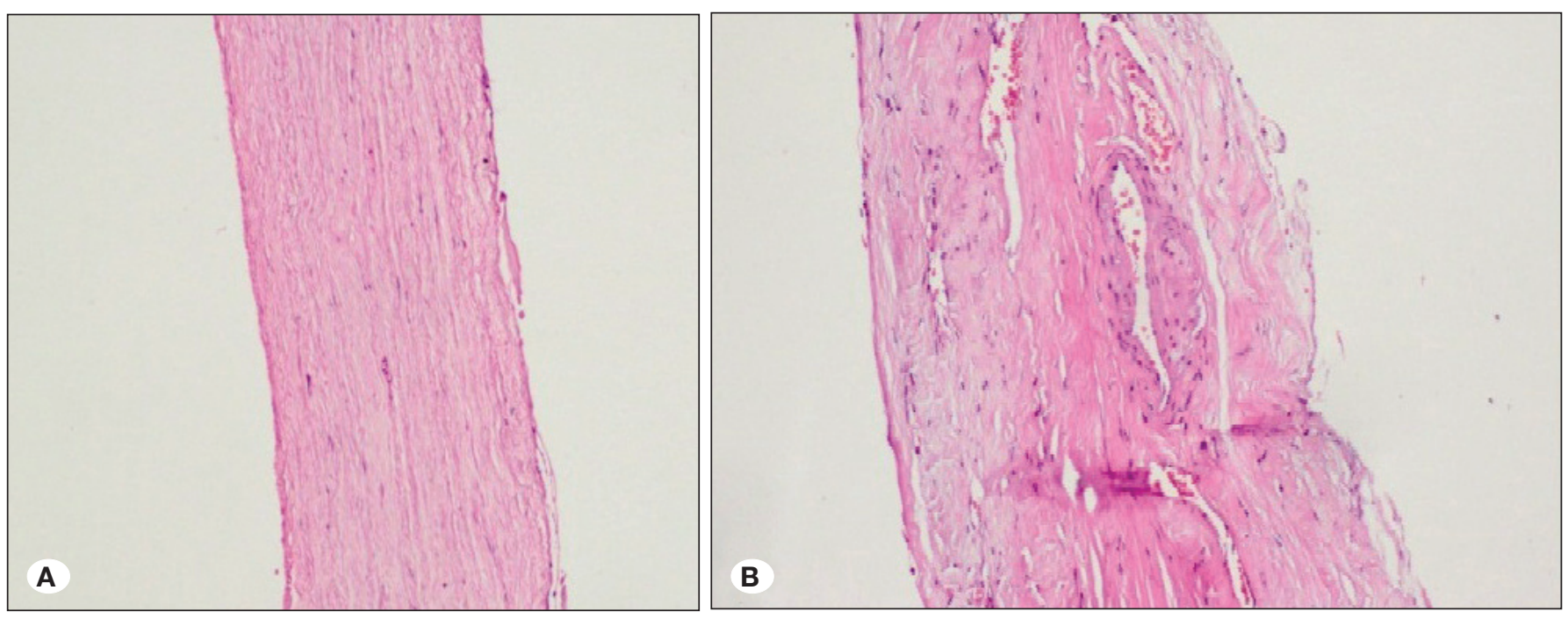

Figure 1: A) The spinal dura comprises a single layer, and the outer and inner faces are lined with a single row of mesothelium. B) The cranial dura comprises the outer dural layer with abundant vessels (the periosteum including fibroblasts and collagen fibers) and the inner dural layer lined with mesothelial cells with connecting firmly composes bilaminar structure in the cranial dura; the mesothelial cells that surround the outside of the dura are not observed (H\&E, $\times 10)$.
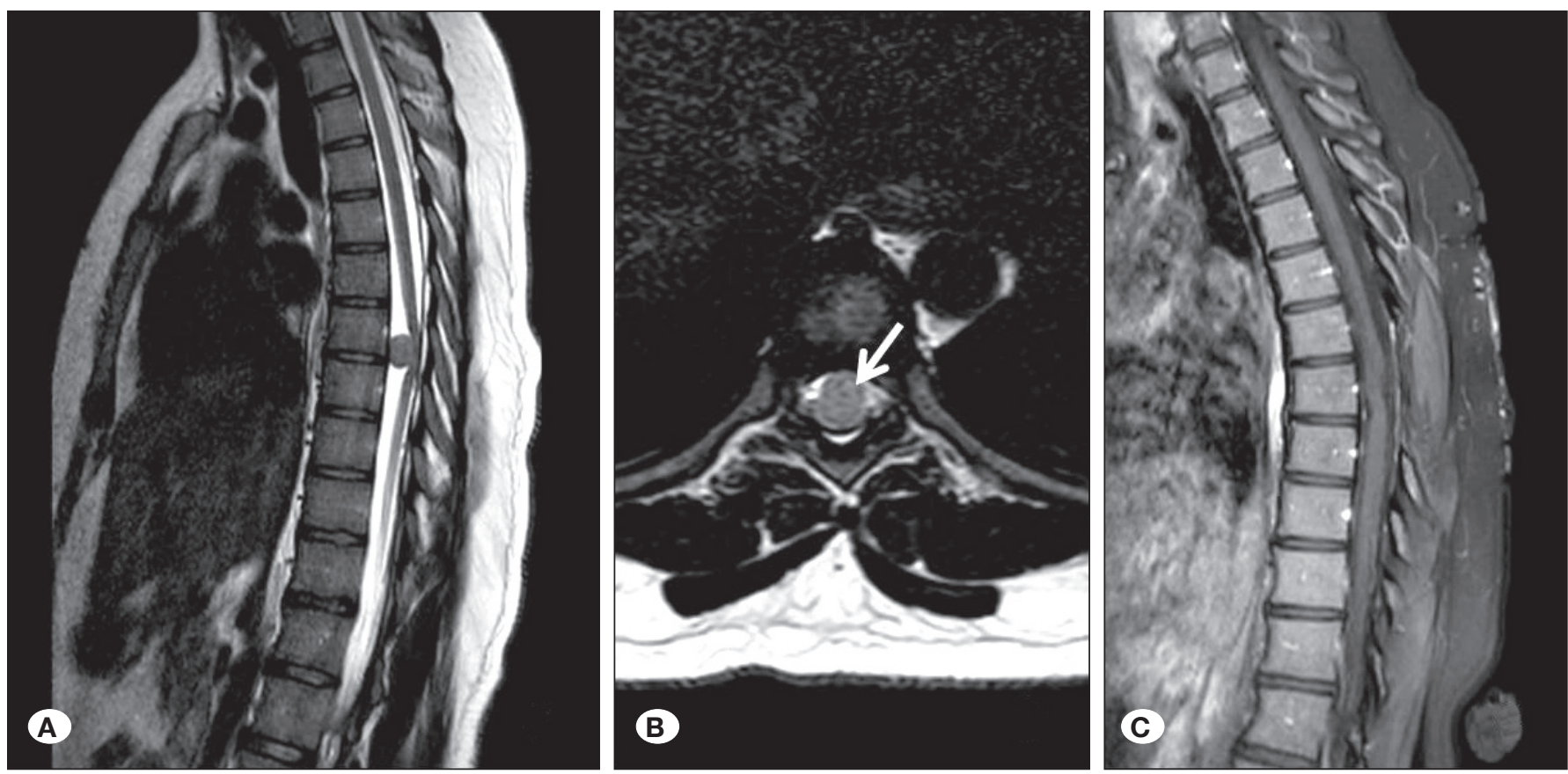

Figure 2: Preoperative sagittal (A) and axial (B) T2-weighted MRI demonstrating a ventrally located meningioma (arrow) posteriorly displacing the spinal cord. Postoperative contrast-enhanced T1-weighted sagittal MRI (C) at 3 years follow-up shows no sign of tumor recurrence. 
is often impossible $(1,3,8,11,18)$. It has been reported that the gross total resection is often sufficient in preventing tumor recurrences $(2,8,11,15,18)$. Nakamura et al. have reported high recurrence rates in cases who underwent Simpson grade 2 resection (10), with recurrence in 6 of 19 cases. They have posited that the presence of residual tumor cells between the inner and outer dural layers might be responsible for high recurrence rate (10). However, such a definition of the spinal dura leads to conceptual confusion. The ultrastructure of the spinal dura, generally described as the outer and inner dural borders, should not be regarded as the outer and inner layers of the cranial dura mater (16). The spinal dura mater is structurally different from the cranial dura mater because the spinal dura mater comprises only one layer (Figure 1A, B). The spinal dura mater forms a loose sheath around the medulla spinalis and represents only the inner or meningeal layer of the cranial dura mater; the outer or endosteal layer ends at the foramen magnum (5). A similar confusion exists in the study by Klekamp and Samii (8), wherein the authors have reported the removal of the inner dural layer. In another study, Saito et al. have reported a new technique for the resection of spinal meningiomas whereby the outer dural layer was preserved (12).

In all our cases, the tumor was totally resected, and the tumor nidus that caused the thickening of the inner dural border was removed by cauterization using bipolar cautery. None of our cases showed recurrence. Furthermore, there was no neurological deterioration due to surgery. Figure $2 \mathrm{~A}-\mathrm{C}$ shows the preoperative and postoperative MRI (at 22 months) findings of case 5. Postoperative CSF fistula developed in one case; however, this complication was not directly associated with the ventral localization of the tumor or with Simpson grade 2 resection. The improvement of symptoms in our cases during the early postoperative period (first 3 months) was remarkable.

The small number of cases may be considered as a limitation of this study. However, we only analyzed cases with ventral localization that underwent Simpson grade 2 resection. When the literature on ventrally located spinal meningiomas with Simpson grade 2 resection is reviewed, the number of patients studied is similar to that in our study $(1-3,6,11,15,17,18)$.

\section{- CONCLUSION}

Simpson grade 2 resection in ventrally located spinal meningiomas results in low complication rates and is satisfactory in terms of recurrence.

\section{- ACKNOWLEDGMENT}

The authors would like to thank Dr. Fatih Serhat EROL for his support and assistance with this study.

\section{- REFERENCES}

1. Arima H, Takami T, Yamagata $T$, Naito K, Abe J, Shimokawa $\mathrm{N}$, Ohata K: Surgical management of spinal meningiomas: A retrospective case analysis based on preoperative surgical grade. Surg Neurol Int 5: 333-338, 2014
2. Boström A, Burgel U, Reinacher P, Krings T, Rohde V, Gilsbach JM, Hans FJ: A less invasive surgical consept for the resection of spinal meningiomas. Acta Neurochir (Wien) 150:551-556, 2008

3. Gezen F, Kahraman S, Canakci Z, Beduk A: Review of 36 cases of spinal cord meningioma. Spine 25:727-731, 2000

4. Gottfried ON, Gluf W, Quinones-Hinojosa A, Kan P, Schmidt $\mathrm{MH}$ : Spinal meningiomas: Surgical management and outcome. Neurosurg Focus 14: e2, 2003

5. Gray H: Anatomy of the Human Body. Philadelphia: Lea \& Febiger, 1918; New York: Bartleby.com, 20th ed, 2000: 551

6. Haegelen C, Morandi X, Riffaud L, Amlashi SFA, Leray E, Brassier G: Results of spinal meningioma surgery in patients with severe preoperative neurological deficits. Eur Spine $J$ 14:440-444, 2005

7. Helseth A, Mork SJ: Primary intraspinal neoplasms in Norway, 1955 to 1986 . A population-based survey of 467 patients. J Neurosurg 71:842-845, 1989

8. Klekamp J, Samii M: Surgical results for spinal meningiomas. Surg Neurol 52:552-562, 1999

9. Levy WJ, Bray J, Dohn D: Spinal cord meningiomas. J Neurosurg 57:804-812, 1982

10. Nakamura M, Tsuji O, Fujiyoshi K, Hosogane N, Watanabe K, Tsuji T, Ishii K, Toyama Y, Chiba K, Matsumoto M: Longterm surgical outcomes of spinal meningiomas. Spine 37:E617-E623, 2012

11. Roux FX, Nataf F, Pineaudeau M, Burne G, Devaux B, Meder JF: Intraspinal meningiomas: Review of 54 cases with discussion of poor prognosis factors and modern therapeutic management. Surg Neurol 46:458-464, 1996

12. Saito T, Arizona T, Maeda T, Terada K, Iwamoto Y: A novel technique for surgical resection of spinal meningioma. Spine 26:1805-1808, 2001

13. Schaller B: Spinal meningioma: Relationship between histological subtypes and surgical outcome? J Neurooncol 75:157-161, 2005

14. Solero CL, Fornari M, Giombini S, Lasio G, Oliveri G, Cimino C, Pluchino F: Spinal meningiomas: Review of 174 operated cases. Neurosurgery 25:153-160, 1989

15. Tsuda K, Akutsu H, Yamamoto T, Nakai K, Ishikawa E, Matsumura A: Is Simpson grade I removal necessary in all cases of spinal meningioma? Assessment of postoperative recurrence during long-term follow-up. Neurol Med Chir (Tokyo) 54: 907-913, 2014

16. Vandenabeele F, Creemers J, Lambrichts I: Ultrastructure of the human spinal arachnoid mater and dura mater. $J$ Anat 189:417-430, 1996

17. Xavier M, Claire H, Laurent R, Seyed A, Mahmoudreza A, Gilles B: Results in the operative treatment of elderly patients with spinal meningiomas. Spine 29:2191-2194, 2004

18. Yoon SH, Chung CK, Jahng TA: Surgical outcome of spinal canal meningiomas. J Korean Neurosurg Soc 42:300-304, 2007 